\title{
X-RAY ECLIPSE DIAGNOSIS OF THE EVOLVING MASS LOSS IN THE RECURRENT NOVA U SCORPII 2010
}

\author{
D. TAKeI ${ }^{1}$, J. J. Drake ${ }^{1}$, M. Tsujimoto ${ }^{2}$, J.-U. Ness ${ }^{3}$, J. P. Osborne ${ }^{4}$, S. Starrfield ${ }^{5}$, and S. Kitamoto ${ }^{6}$ \\ ${ }^{1}$ Smithsonian Astrophysical Observatory, 60 Garden Street, Cambridge, MA 02138, USA; dtakei@ head.cfa.harvard.edu \\ 2 Japan Aerospace Exploration Agency, Institute of Space and Astronautical Science, 3-1-1 Yoshino-dai, Chuo-ku, Sagamihara, Kanagawa 252-5210, Japan \\ ${ }^{3}$ European Space Agency, XMM-Newton Observatory SOC, SRE-OAX, Apartado 78, E-28691 Villanueva de la Cañada, Madrid, Spain \\ ${ }^{4}$ Department of Physics and Astronomy, University of Leicester, Leicester LE1 7RH, UK \\ ${ }^{5}$ School of Earth and Space Exploration, Arizona State University, Tempe, AZ 85287-1404, USA \\ ${ }^{6}$ Department of Physics, Rikkyo University, 3-34-1 Nishi-Ikebukuro, Toshima, Tokyo 171-8501, Japan \\ Received 2013 January 11; accepted 2013 March 20; published 2013 May 2
}

\begin{abstract}
We report the Suzaku detection of the earliest X-ray eclipse seen in the recurrent nova U Scorpii 2010. A targetof-opportunity observation 15 days after the outburst found a $27 \% \pm 5 \%$ dimming in the $0.2-1.0 \mathrm{keV}$ energy band at the predicted center of an eclipse. In comparison with the X-ray eclipse depths seen at two later epochs by XMM-Newton, the source region shrank by about $10 \%-20 \%$ between days 15 and 35 after the outburst. The X-ray eclipses appear to be deeper than or similar to contemporaneous optical eclipses, suggesting the X-ray and optical source region extents are comparable on day 15 . We raise the possibility of the energy dependency in the photon escape regions, and that this would be a result of the supersoft X-ray opacity being higher than the Thomson scattering opacity at the photosphere due to bound-free transitions in abundant metals that are not fully ionized. Assuming a spherically symmetric model, we constrain the mass-loss rate as a function of time. For a ratio of actual to Thomson opacity of 10-100 in supersoft X-rays, we find an ejecta mass of about $10^{-7}-10^{-6} M_{\odot}$.
\end{abstract}

Key words: novae, cataclysmic variables - stars: individual (U Scorpii) - X-rays: stars

Online-only material: color figures

\section{INTRODUCTION}

A nova explosion occurs in an accreting binary system comprising a white dwarf and a red dwarf companion. When the amount of accreted material reaches a critical mass, hydrogen fusion is triggered by a thermonuclear runaway on the white dwarf (Starrfield et al. 2008). An event is mainly characterized by the development of photospheric emission powered by nuclear burning after the explosion. This is the first instance in the optical where a sudden increase in brightness is the result of radiative transfer through the optically thick ejecta. As the ejecta expand and become less opaque, the dominant emission shifts toward higher energies, eventually becoming supersoft $X$ rays. The spectral hardening occurs because the mass outflow diminishes with time and consequently the pseudo-photosphere is formed deeper in the expanding ejecta (e.g., Bath 1978). For detailed reviews, see, e.g., Warner (2003) and Bode \& Evans (2008).

Our understanding of nova evolution to date has almost entirely resulted from photometric and spectroscopic studies. While all novae are thought to be binaries, and some fraction will inevitably be eclipsing, the utilization of eclipses during an explosion as a powerful and direct probe of the emitting geometry has not yet been fully realized. Observing such a phenomenon is difficult for most novae because they spend many thousands of years between outbursts and are therefore observed only once, with no prior information on the nature of the progenitor. However, several novae have been observed to explode more than once in a human lifetime and are called recurrent novae. Since they can be studied in detail prior to an eruption, they are invaluable for understanding the nature of novae and cataclysmic variables.

In this Letter, we investigate the development of the supersoft source (SSS) region for the eclipsing recurrent nova U Scorpii 2010. Using the Suzaku satellite we have detected a dimming
15 days after the outburst, corresponding to the earliest postoutburst X-ray eclipse ever reported for this object. Through comparison with a $30 \%-50 \%$ dimming in X-ray eclipses at two subsequent epochs observed by XMM-Newton (Ness et al. 2012), we find that the X-ray source shrank with time. For the first time we are able to use direct geometric information provided by supersoft $X$-ray eclipses to investigate the evolution of the radiatively driven flow in the early stage of an explosion.

\section{TARGET (U SCORPII)}

U Scorpii is an eclipsing binary undergoing nova explosions recurrently about every 10 years. Novae have been observed 10 times, the most recent of which were in 1999 and 2010 (Schaefer 2010a). Starrfield et al. (1988) noted that the short time between outbursts points to a mass-gaining white dwarf close to the Chandrasekhar limit. The system has an inclination $\sim 80^{\circ}$, and the radii of the companion star and the binary orbit are 2.7 and $6.9 R_{\odot}$, respectively, according to Hachisu et al. (2000a), and are 2.1 and $6.5 R_{\odot}$ by Thoroughgood et al. (2001). The orbital period before the 1999 outburst was 1.2305521 days (Schaefer \& Ringwald 1995), while the period from 2001 to 2009 was 1.23054695 days (Schaefer et al. 2010c), indicating that it changed due to the 1999 eruption (Matsumoto et al. 2003). We adopt the Schaefer et al. (2010c) ephemeris with an origin of HJD 2,451,234.539. An optical eclipse covers $\sim 18 \%$ of an orbital phase in the quiescent state, which corresponds to 0.22 days (Schaefer et al. 2010c).

The tenth observed eruption was discovered on 2010 January 28.4385 UT (MJD 55,224.4385) by B. G. Harris (Schaefer et al. 2010a). We define the epoch of the discovery as the origin of time. A worldwide collaboration was organized, and subsequent studies were conducted by ground- and space-based telescopes (e.g., Osborne et al. 2010; Schaefer 2010b). The development of optical and X-ray brightness is shown in Figure 1. Optical 


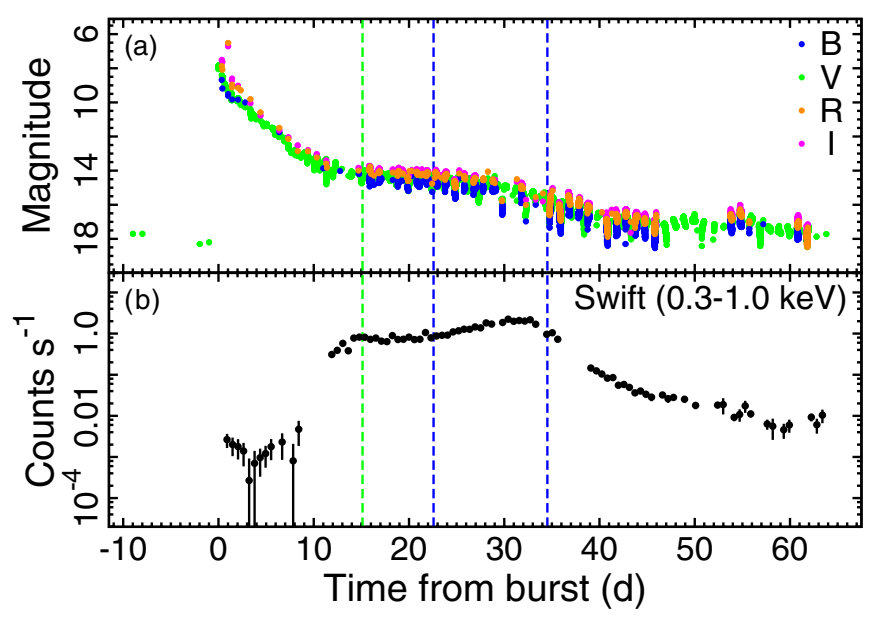

Figure 1. Development of (a) optical and (b) X-ray brightness in the nova outburst of U Scorpii 2010. The origin of the abscissa is MJD 55224.4385 when the nova was discovered (Schaefer et al. 2010a). The times of the Suzaku and $X M M-N e w t o n$ observations are shown by vertical dashed lines in green and blue, respectively. (a) Optical magnitudes are from the American Association of Variable Star Observers (AAVSO), the Variable Star Observers League in Japan (VSOLJ), and Table 2 in Schaefer et al. (2011). (b) Background-subtracted count rates of the Swift (Schaefer et al. 2010b) in the 0.3-1.0 keV energy band. (A color version of this figure is available in the online journal.)

eclipses imply that the source region was $4.1,3.4$, and 2.2 $R_{\odot}$ on days $15-26,26-41$, and 41-67, respectively (Schaefer et al. 2011). The eclipse shapes were consistent with a spherical source until day 26 , with apparently more disk-like morphology at later times. A $30 \%-50 \%$ dimming in X-rays during expected times of eclipse were found by XMM-Newton on days 23 and 35 (Ness et al. 2012), suggesting that the size of the X-ray source was comparable to the optical and orbital sizes. The X-ray light curve on day 23 exhibited oscillations, which Ness et al. (2012) interpreted in terms of a reforming accretion disk.

\section{OBSERVATIONS AND REDUCTION}

We performed target-of-opportunity observations of $U$ Scorpii on 2010 February 6, 9, and 12 (9, 12, and 15 days after the outburst, respectively) with the Suzaku X-ray satellite. Suzaku (Mitsuda et al. 2007) has an X-ray Imaging Spectrometer (XIS; Koyama et al. 2007) and a Hard X-ray Detector (HXD; Takahashi et al. 2007; Kokubun et al. 2007). We concentrate on the XIS data on day 15 that show a clear eclipse. The day 9 data are poor in statistical quality, and the light curve on day 12 exhibited substantial stochastic variability that prevented unambiguous measurement of the eclipse signature.

The XIS is equipped with four X-ray CCDs at the foci of four co-aligned X-ray telescope modules (Serlemitsos et al. 2007). Three of them (XIS0, 2, and 3) are front-illuminated CCDs sensitive in the 0.4-12 keV energy band, while the remaining one (XIS1) is a back-illuminated CCD sensitive in the range $0.2-12 \mathrm{keV}$. XIS2 and a part of XIS0 are not functional and their data were excluded. The XIS was operated in the normal clocking mode with an $8 \mathrm{~s}$ frame time.

Data were processed with pipeline version 2.4.12.27. Events were removed during South Atlantic anomaly passages, when night-earth elevation angles were below $5^{\circ}$, and day-earth elevation angles were below $20^{\circ}$. The net exposure time is $27 \mathrm{ks}$. For data reduction, we used the HEASoft package version 6.10 and the calibration database version xis20090925/xrt20080709.

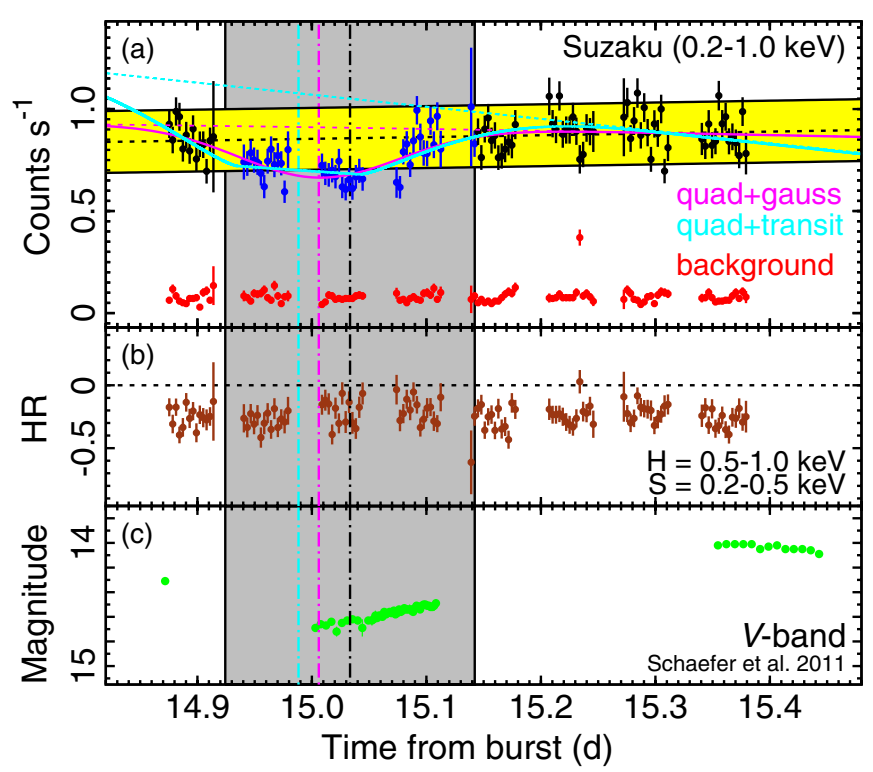

Figure 2. (a) XIS $0.2-1.0 \mathrm{keV}$ count rates in eclipse (blue) and at other times (black), together with the background (red). The time of the optical eclipse is illustrated by the gray shaded region and the black dashed-dotted line. The bestfit quadratic model applied to the data outside eclipse is represented by a black dashed line. The yellow region shows the $\pm 90 \%$ range of the black data points. The best-fit eclipse models are shown color-coded by solid lines; the quadratic component is illustrated by the dashed lines. The vertical dashed-dotted lines in magenta and cyan indicate the Gaussian and transit center, respectively. (b) The hardness ratios (HRs) defined by $(H-S) /(H+S)$, where $H$ and $S$ are rates in the $0.5-1.0 \mathrm{keV}$ and $0.2-0.5 \mathrm{keV}$ energy bands, respectively. (c) The $V$-band magnitudes from Schaefer et al. (2011).

(A color version of this figure is available in the online journal.)

\section{ANALYSIS}

X-ray light curves and spectra were constructed by taking source events accumulated from a circular region of 130 pixels radius (2!3) adaptively chosen from XIS images to maximize the signal-to-noise ratio. Barycentric correction of photon arrival times was applied. Events taken with the three XIS were merged, and background was estimated from an annular region with inner and outer radii of 180 and 250 pixels (3'1-4'.2), respectively. The photon pile-up fraction was $\lesssim 0.1 \%$. Simple spectral fitting of the extracted photon events on day 15 yielded similar model parameters (blackbody temperature $\sim 24 \mathrm{eV}$; bremsstrahlung temperature $\sim 0.7 \mathrm{keV}$ ) to those obtained by Ness et al. (2012) for the day $23 X M M-N e w t o n$ spectrum, indicating only slow spectral evolution through this period. The SSS component dominates the X-ray spectrum, in which the bremsstrahlung component contributes at most only $\sim 1 \%$ of the total flux and/or count rates in the $0.2-1.0 \mathrm{keV}$ energy band.

Effective areas were computed by incorporating the history of the satellite aspect solution using a Monte Carlo simulation tool (xissim; Ishisaki et al. 2007) that also accounts for an aspectrelated vignetting of count rates by $10 \%-20 \%$ due to the loss of a gyro system on Suzaku. Finally, all count rates were normalized to values corresponding to extraction from a circular aperture of $3^{\prime}$ radius. The resulting background-subtracted light curve and hardness ratios in the $0.2-1.0 \mathrm{keV}$ energy band, together with the $V$-band magnitudes from Schaefer et al. (2011), are shown in Figure 2.

A shallow dip in the X-ray light curve is coincident with the predicted optical eclipse on day 15 (Figure 2(a)). Comparison of the distribution of counts during the optical eclipse with those outside of eclipse yields a null hypothesis probability that the 
$\mathrm{X}$-ray dimming is explained by a random source fluctuation on top of the global trend of $<0.01$. No significant correlation was found between the flux variation and the spectral hardness (Figure 2(b)), suggesting that the variation of intrinsic colors does not affect the dimming. We thus conclude that the X-ray dimming is due to the binary eclipse.

The X-ray eclipse was measured by fitting the entire light curve using a quadratic function plus a Gaussian component to represent the source emission and approximate the eclipse dimming, respectively (Figure 2(a)). The model yielded an eclipse depth of $27 \% \pm 5 \%$ at a center of $15.006 \pm$ 0.012 days after the outburst with a duration of $0.17 \pm 0.04$ days $(0.13 \pm 0.03$ orbital phase) in the FWHM ( $1 \sigma$ errors $)$. The eclipse center was shifted by $\sim 0.03$ days in comparison with the optical ephemeris of Schaefer et al. (2010c). At face value this is a significant offset. We note, however, that the optical eclipse minima observed by Schaefer et al. (2011) show jitter of a similar magnitude. This jitter is likely due to small fluctuating brightness inhomogeneities and departures from perfect sphericity in the emitting region.

\section{DISCUSSION}

\subsection{Source Emitting Region}

The X-ray eclipse provides a means for constraining the geometry of the emitting region. Assuming a spherically symmetric source with no limb-darkening and the system parameters of Hachisu et al. (2000a), the $27 \% \pm 5 \%$ dimming on day 15 implies that the X-ray source had roughly four times the area and twice the radius of the companion star, corresponding to a source radius of $5.1 \pm 0.6 R_{\odot}$. This is consistent with a fit to the light curve using a quadratic function and analytic transit model (Mandel \& Agol 2002) assuming the same geometry, for which we obtained a radius $4.5 \pm 0.2 R_{\odot}$ and center epoch $14.988 \pm 0.007$ days post-outburst; see Figure 2(a). By adopting the slightly more compact system parameters of Thoroughgood et al. (2001), radius estimates are smaller by a factor of $\sim 0.8$. This emitting region within the residual outflow is large in comparison to the underlying white dwarf and was referred to as a "corona" by Ness et al. (2012).

The development of the source region can be studied by comparing the above radii with the XMM-Newton results taken at two different epochs (Ness et al. 2012). The X-ray light curve on day 23 exhibited a dimming of up to $\sim 50 \%$ with $\sim 20 \%$ oscillations during an eclipse. The latter could be caused by absorption in a reforming accretion disk (Ness et al. 2012), or by the geometrical eclipse of a photometrically varying photon escape region caused by inhomogeneities and/or density instabilities in the radiatively driven flow (e.g., Shaviv \& Dotan 2010; Shaviv 2005; Owocki et al. 1988). In contrast, the $\mathrm{X}$-ray light curve on day 35 showed a clear eclipse with a $\sim 40 \%$ dimming, corresponding to a source radius of $\sim 4.2 R_{\odot}$, indicating that the X-ray source shrunk by about $10 \%-20 \%$ between 15 and 35 days after the outburst. This is the first quantitative X-ray measurement of the shrinking of the source radius of an emerging SSS in a nova outburst.

The standard nova paradigm posits that the SSS emission emerges as ejecta expand and decline in the radiatively driven flow allows photon escape at successively smaller radial distance from a white dwarf surface until supersoft X-ray temperatures are reached. Once this begins and the outer ejecta are thin, the source radius is of the order of a few solar radii or smaller and the atmosphere is possibly puffed-up and porous (e.g.,
Shaviv \& Dotan 2010). This allows it to be much larger than the Eddington luminosity and X-ray temperature would otherwise suggest (e.g., Shaviv 2005). A few thousand $\mathrm{km} \mathrm{s}^{-1}$ outflow traverses the source in only $\sim 100 \mathrm{~s}$; at this point, any further change in radius probes the instantaneous mass-loss rate in the outflow. We investigate this below, first assuming the simplest ejecta model, and then examining the implications of an energydependent opacity by comparing source radii in optical and $\mathrm{X}$-rays on day 15 .

\subsection{Simple Ejecta Model}

Assuming a spherically symmetric flow from the white dwarf surface, the X-ray photon escape radius $R_{x}$ is related to the optical depth by

$$
\tau=\int_{R_{x}}^{R_{\text {out }}} \kappa \rho d r,
$$

where $R_{\text {out }}$ is the outermost radius of the ejecta, $\kappa$ is the ejecta opacity, and $\rho$ is the density as a function of radial distance $r$. We define the X-ray photon escape layer as a point at which $\tau$ is approximately unity. Assuming that the ejected gases are accelerated within a small radial distance and that the terminal velocity $v_{\infty}$ has not changed appreciably since the eruption (i.e., the timescale of the terminal velocity decline is much larger than the time for the ejecta to reach $\left.R_{\text {out }}\right), R_{\text {out }} \approx v_{\infty} t$. We adopt $v_{\infty}=$ $3000 \mathrm{~km} \mathrm{~s}^{-1}$ from the estimate of Yamanaka et al. (2010) based on $\mathrm{H} \alpha$ line profiles, and $\kappa=0.4 \mathrm{~cm}^{2} \mathrm{~g}^{-1}$ as the Thomson scattering opacity in a fully ionized hydrogen-dominated gas. The radial density profile is given by

$$
\rho=\frac{1}{4 \pi r^{2}} \frac{\dot{M}}{v_{\infty}},
$$

where $\dot{M}$ is the mass-loss rate. We next assume a time-dependent mass-loss rate from the white dwarf given by (e.g., Bode \& Evans 2008)

$$
\dot{M}=\dot{M}_{0}\left(t_{0} / t\right)^{p},
$$

where $\dot{M}_{0}$ is an initial value normalized at time $t=t_{0}=1 \mathrm{~s}$ and $p$ is a measure of the speed of its decline. Using these relations, we fitted the observed radii of the X-ray source regions at 3 epochs (days 15 (Suzaku), 23 and 35 (XMMNewton)) with 2 free parameters $\dot{M}_{0}$ and $p$. We used the Gaussian estimate with the Hachisu et al. (2000a) parameters on day 15 in order to compare directly the eclipse depths with the other epochs. We further adopted $10 \%$ uncertainties on the source radii derived by XMM-Newton based on the light curves in Ness et al. (2012). The source radius on day 23 depends on the interpretation of the light-curve oscillations, thus both cases of $30 \%$ and $50 \%$ eclipse dimming (cases 1 and 2, respectively) were treated separately. The best-fit results and $1 \sigma$ confidence regions are shown in Figure 3, where $\dot{M}_{0}=1 \times 10^{-3}$ $\left(6 \times 10^{-5}-3 \times 10^{-2}\right) M_{\odot} \mathrm{yr}^{-1}$ and $p=0.23 \pm 0.22$ in case 1 ; $\dot{M}_{0}=5 \times 10^{-3}\left(3 \times 10^{-4}-2 \times 10^{-1}\right) M_{\odot} \mathrm{yr}^{-1}$ and $p=0.33 \pm$ 0.25 in case 2 .

Integration over the time-dependent mass-loss rate yields a rough estimate of the total ejecta mass $M_{\mathrm{ej}}$ as a function of elapsed time and is shown in Figure 3. Assuming that the SSS faded and the mass outflow was terminated on day 40 (Figure 1(b)), the total ejecta mass is about 5-8 $\times 10^{-6} M_{\odot}$ for the simple model. This is consistent with the changing orbital period from long-term optical monitoring $\left(4.3 \pm 6.7 \times 10^{-6} M_{\odot}\right.$; Schaefer 2011), but slightly higher than theoretical estimates for 


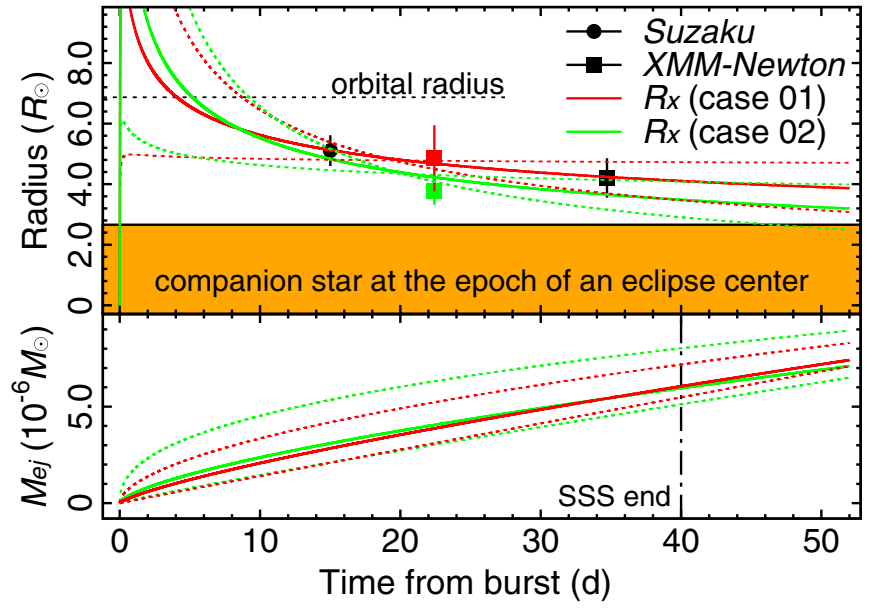

Figure 3. Top: the best-fit source radius model vs. time (solid) and $1 \sigma$ confidence (dashed) for radii inferred from Suzaku and XMM-Newton eclipses. Models for day 23 eclipse depths of $30 \%$ (red, case 1) and 50\% (green, case 2) are shown separately. The orange region and the dashed line indicate the sizes of the companion star and the orbital separation, respectively. Bottom: total ejected mass vs. time corresponding to the upper panel models. The vertical dasheddotted line shows the end of the SSS phase.

(A color version of this figure is available in the online journal.)

U Scorpii (e.g., $4 \times 10^{-7} M_{\odot}$ : Starrfield et al. $1988 ; 2 \times 10^{-6} M_{\odot}$ : Hachisu et al. 2000b). We here note that mass-related values also become smaller by a factor of $\sim 0.8$ by adopting Thoroughgood et al. (2001) parameters.

\subsection{Optical versus $X$-Ray Eclipses}

Schaefer et al. (2011) found that the eclipse shapes in optical were consistent with a spherical source with a radius of $4.1 R_{\odot}$ on days 15-26. Since we have assumed spherically symmetric source regions whereas the optical eclipses (Schaefer et al. 2011) and the day 23 XMM-Newton eclipses (Ness et al. 2012) were accompanied by more complicated structure, we cannot conclude with certainty that there is a significant difference between the sizes of the optical and X-ray photon escape regions, though at face value the different inferred radii suggest this on day 15 with the system parameters of Hachisu et al. (2000a).

A remaining puzzle is the origin of the optical plateau started from $\sim 10$ to 15 days post-outburst. This optical flux clearly exceeds the extrapolated X-ray blackbody with the Eddington luminosity of a Chandrasekhar white dwarf, though a blackbody is grossly inadequate to describe the true spectrum and the magnitude of any optical excess remains uncertain. Hachisu et al. (2000a) argued that the optical flux included reprocessing photospheric emission on the surface of the reappearing accretion disk. Currently, no clear evidence is found for this because it is unknown how early the accretion process resumes after the explosion, though recent studies imply that the non-accretion case is more likely, at least on day 15. Simulations of Drake \& Orlando (2010) found that the accretion disk was completely destroyed by the blast. Mason et al. (2012) and Worters et al. (2010) suggest resumption of accretion based on spectroscopy and optical flickering, respectively, around day 8, though the shortterm variability disappeared on day 15 (Munari et al. 2010). Schaefer et al. (2011) found the optical source spherical until day 26 based on eclipse mapping. Ness et al. (2012) further discussed reforming accretion still on day 23. Alternatively, the reprocessing might occur in surrounding ejecta with a substan- tial optical depth in supersoft X-rays, though it has to be noted that any scenario at this time remains largely speculative.

If we assume that the optical flux on day 15 originated mostly from the surrounding ejecta, the possible difference between the radii of optical $\left(R_{o} \sim 4 R_{\odot}\right)$ and X-ray $\left(R_{x} \sim\right.$ $5 R_{\odot}$ ) sources can arise from the energy dependence of the photon escape radii that would result from different optical and X-ray opacities ( $\kappa_{o}$ and $\kappa_{x}$, respectively). For a plasma at temperatures of $10-100 \mathrm{eV}$, the opacity in the supersoft $\mathrm{X}$-ray range can be significantly larger than the Thomson crosssection owing to abundant elements (primarily $\mathrm{C}, \mathrm{N}, \mathrm{O}$, and $\mathrm{Ne}$ ) not being fully ionized. For a low-density plasma, the optical opacity will instead be close to the Thomson value. Using the PINTofALE $^{7}$ routine IONABS (Kashyap \& Drake 2000), we computed the cross-section assuming collisional equilibrium at the temperature of $50 \mathrm{eV}$ and found $\kappa_{x} \sim 50 \mathrm{~cm}^{2} \mathrm{~g}^{-1}$ in the $0.2-1.0 \mathrm{keV}$ range, or $\sim 100$ times the Thomson value. The plasma in the ejecta is in fact likely to be photoionized rather than in collisional equilibrium. For a constant wind velocity, Equations (1) and (2) imply for optical depth unity $\kappa \rho r \sim 1$, or $\rho \sim 5 \times 10^{12} \mathrm{H}$ atoms $\mathrm{cm}^{-3}$ at $4 R_{\odot}$. The ionization parameter, $\xi=L / n_{e} r^{2}$ (e.g., Tarter et al. 1969), is then of the order of $10^{2}$ for the Eddington luminosity and indicates the outflow is photon-dominated. Nevertheless, our cross-section estimate suggests that the true supersoft X-ray opacity could be an order of magnitude or more larger than that in the optical.

The density profile, and consequently the mass-loss rate and total mass loss, derived from the X-ray eclipses scales inversely with the gas opacity, so that the total mass-loss rate would be $5-8 \times 10^{-6}\left(\kappa_{o} / \kappa_{x}\right) M_{\odot}$. We have argued that the $\mathrm{X}$-ray opacity at the photosphere is higher than the Thomson value that characterizes the optical range, and that this would give rise to the observed difference in optical and X-ray source radii. Theoretical mass-loss estimates are in the range $4 \times 10^{-7}-2 \times 10^{-6} M_{\odot}$ (Starrfield et al. 1988; Hachisu et al. $2000 \mathrm{~b}$ ), indicating the true X-ray opacity might be higher than the optical one by a factor of about 2-20. Drake \& Orlando (2010) found the initial explosion threw off probably no more than $10^{-7} M_{\odot}$ using non-spherical hydrodynamic models and early X-ray luminosity constraints. Estimates of the subsequent mass loss then imply that most of the mass was lost during later evolution, confirming theoretical expectations (e.g., Gallagher \& Starrfield 1978).

We thank Suzaku for undertaking our target-of-opportunity program, and B. E. Schaefer for organizing the U Scorpii 2010 collaboration. Optical data are from AAVSO and VSOLJ databases. We acknowledge support from the JSPS (D.T.), NASA contract NAS8-03060 to the CXC (J.J.D.), the UK Space Agency (J.O.), and NSF and NASA grants to ASU (S.S.).

\section{REFERENCES}

Bath, G. T. 1978, MNRAS, 182, 35

Bode, M. F., \& Evans, A. 2008, Classical Novae, ed. M. F. Bode \& A. Evans (Cambridge: Cambridge Univ. Press)

Drake, J. J., \& Orlando, S. 2010, ApJL, 720, L195

Gallagher, J. S., \& Starrfield, S. 1978, ARA\&A, 16, 171

Hachisu, I., Kato, M., Kato, T., \& Matsumoto, K. 2000a, ApJL, 528, L97

Hachisu, I., Kato, M., Kato, T., Matsumoto, K., \& Nomoto, K. 2000b, ApJL, 534, L189

Ishisaki, Y., Maeda, Y., Fujimoto, R., et al. 2007, PASJ, 59, S113

Kashyap, V., \& Drake, J. J. 2000, BASI, 28, 475

http://hea-www.harvard.edu/PINTofALE/ 
Kokubun, M., Makishima, K., Takahashi, T., et al. 2007, PASJ, 59, S53 Koyama, K., Tsunemi, H., Dotani, T., et al. 2007, PASJ, 59, S23

Mandel, K., \& Agol, E. 2002, ApJL, 580, L171

Mason, E., Ederoclite, A., Williams, R. E., Della Valle, M., \& Setiawan, J. 2012, A\&A, 544, A149

Matsumoto, K., Kato, T., \& Hachisu, I. 2003, PASJ, 55, 297

Mitsuda, K., Bautz, M., Inoue, H., et al. 2007, PASJ, 59, S1

Munari, U., Dallaporta, S., \& Castellani, F. 2010, IBVS, 5930, 1

Ness, J.-U., Schaefer, B. E., Dobrotka, A., et al. 2012, ApJ, 745, 43

Osborne, J. P., Page, K. L., Wynn, G., et al. 2010, ATel, 2442,

Owocki, S. P., Castor, J. I., \& Rybicki, G. B. 1988, ApJ, 335, 914

Schaefer, B. E. 2010a, ApJS, 187, 275

Schaefer, B. E. 2010b, arXiv:1009.3197

Schaefer, B. E. 2011, ApJ, 742, 112

Schaefer, B. E., Harris, B. G., Dvorak, S., Templeton, M., \& Linnolt, M. 2010a, IAUC, 9111, 1

Schaefer, B. E., Pagnotta, A., LaCluyze, A. P., et al. 2011, ApJ, 742, 113

Schaefer, B. E., Pagnotta, A., Osborne, J. P., et al. 2010b, ATel, 2477, 1
Schaefer, B. E., Pagnotta, A., Xiao, L., et al. 2010c, AJ, 140, 925

Schaefer, B. E., \& Ringwald, F. A. 1995, ApJL, 447, L45

Serlemitsos, P. J., Soong, Y., Chan, K., et al. 2007, PASJ, 59, S9

Shaviv, N. J. 2005, in ASP Conf. Ser. 332, The Fate of the Most Massive Stars, ed. R. Humphreys \& K. Stanek (San Francisco, CA: ASP), 180

Shaviv, N. J., \& Dotan, C. 2010, MmSAI, 81, 350

Starrfield, J. H., Illiadis, C., \& Hix, W. R. 2008, in Classical Novae, ed. M. Bode \& A. Evans (Cambridge: Cambridge Univ. Press), 77

Starrfield, S., Sparks, W. M., \& Shaviv, G. 1988, ApJL, 325, L35

Takahashi, T., Abe, K., Endo, M., et al. 2007, PASJ, 59, S35

Tarter, C. B., Tucker, W. H., \& Salpeter, E. E. 1969, ApJ, 156, 943

Thoroughgood, T. D., Dhillon, V. S., Littlefair, S. P., Marsh, T. R., \& Smith, D. A. 2001, MNRAS, 327, 1323

Warner, B. (ed.) 2003, Cataclysmic Variable Stars (Cambridge: Cambridge Univ. Press)

Worters, H. L., Eyres, S. P. S., Rushton, M. T., \& Schaefer, B. 2010, IAUC, 9114, 1

Yamanaka, M., Uemura, M., Kawabata, K. S., et al. 2010, PASJ, 62, L37 